\title{
Calves are susceptible to infection with the newly emerged porcine deltacoronavirus, but not with the swine enteric alphacoronavirus, porcine epidemic diarrhea virus
}

\author{
Kwonil Jung ${ }^{1} \cdot$ Hui Hu${ }^{1,2} \cdot$ Linda J. Saif ${ }^{1}$
}

Received: 22 December 2016/Accepted: 18 February 2017/Published online: 3 April 2017

(C) Springer-Verlag Wien 2017

\begin{abstract}
Fecal virus shedding, seroconversion and histopathology were evaluated in 3-7-year-old gnotobiotic calves orally inoculated with porcine deltacoronavirus (PDCoV) (9.0-9.6 $\log _{10}$ genomic equivalents [GE] of OHFD22-P5; $n=4)$ or porcine epidemic diarrhea virus (PEDV) (10.2-12.5 $\log _{10}$ GE of PC21A; $\left.n=3\right)$. In PDCoV-inoculated calves, an acute but persisting fecal viral RNA shedding and PDCoV-specific serum IgG antibody responses were observed, but without lesions or clinical disease. However, no fecal shedding, seroconversion, histological lesions, and clinical disease were detected in PEDV-inoculated calves. Our data indicate that calves are susceptible to infection by the newly emerged PDCoV, but not by the swine coronavirus, PEDV.
\end{abstract}

Coronaviruses (CoVs) are enveloped, single-stranded RNA viruses of positive-sense polarity. Their genomes range from approximately 26 to $32 \mathrm{~kb}$ in size [16]. The family Coronaviridae of the order Nidovirales is divided into the four genera: Alphacoronavirus, Betacoronavirus, Gammacoronavirus, and Deltacoronavirus. Bats are the projected host reservoir for alphacoronaviruses and

Kwonil Jung

jung.221@osu.edu

$\triangle$ Linda J. Saif

saif.2@osu.edu

1 Food Animal Health Research Program, Ohio Agricultural Research and Development Center, Department of Veterinary Preventive Medicine, The Ohio State University, 1680 Madison Ave., Wooster, OH 44691, USA

2 College of Animal Science and Veterinary Medicine, Henan Agricultural University, Zhengzhou, China betacoronaviruses, while birds are thought to be the host for gammacoronaviruses and deltacoronaviruses [18]. The two betacoronaviruses, severe acute respiratory syndrome $\mathrm{CoV}$ (SARS-CoV) and Middle East respiratory syndrome $\mathrm{CoV}$ (MERS-CoV), were transmitted by civet cats and camels, respectively, to humans, but they have shown limited capacity for adaptation to humans [16]. Some CoVs can be transmitted to different animal species, and subsequently adapt to and be maintained in the new host, because they can exploit or share a variety of host cell surface molecules or other undefined factors [16].

The species Porcine epidemic diarrhea virus belongs to the genus Alphacoronavirus. Porcine epidemic diarrhea virus (PEDV) causes acute diarrhea, dehydration and high mortality in neonatal piglets [9]. For the last four decades, since the first appearance of PEDV in 1977, PEDV infection has resulted in significant economic losses in the European, Asian and US swine industries. PEDV has been found only within the pig population, indicating that the virus might have adapted only to pigs [9]. The species Porcine deltacoronavirus belongs to the genus Deltacoronavirus. Porcine deltavoronavirus (PDCoV) is a novel enteropathogenic $\mathrm{CoV}$ infecting pigs and was previously reported in birds [10]. PDCoV was first identified in pigs in Hong Kong in 2012 [18] and the associated enteric disease was first reported in US swine only in early 2014 [17]. However, the origin of PDCoV infection in pigs and also the sudden emergence and route of introduction of this virus in the US remains unclear [10]. PDCoV may have only partially adapted to pigs and still retain some potential to infect different animal species, particularly poultry or other livestock, which can often have frequent contact with pigs in small-scale, backyard farms in the US. Therefore, our aim was to determine whether calves are susceptible to infection with either the newly emerged PDCoV or the 
swine enteric CoV, PEDV. Fecal virus shedding, seroconversion and histopathology were evaluated in gnotobiotic (Gn) calves orally inoculated with PDCoV or PEDV.

The PDCoV OH-FD22 virus was isolated and then serially passaged five times (P5) in LLC porcine kidney (LLC-PK) cells (ATCC CL-101) [2]. The virus was orally inoculated and propagated in a 9-day-old $\mathrm{Gn}$ pig. The viral RNA titer of OH-FD22-P5 used as inoculum in the intestinal contents (ICs) was $9.0 \log _{10}$ genomic equivalents (GE)/ml. The wild-type US PEDV strain PC21A, propagated in a Gn pig [7], was also used in this study. All ICs were negative for other enteric viruses, such as rotavirus groups A-C, by PCR/RT-PCR [6].

Near-term Angus $\times$ Jersey crossbred Gn calves were delivered aseptically by caesarean section [5]. Eight 3- to 7-day-old calves were randomly assigned to three groups: PDCoV infection ( $n=4$; calves \#1-4), PEDV infection ( $n=3$; calves \#5-7), and mock (minimum essential medium [MEM]; $n=1$; calf \#8, 3 days of age) (Table 1). Calves \#1-4 were inoculated orally with 9.0-9.6 $\log _{10}$ GE of the OHFD22-P5, and calves \#5-7 were inoculated orally with 10.2-12.5 $\log _{10}$ GE of the PC21A (Table 1). After viral inoculation, we monitored clinical signs daily. Diarrhea was assessed by scoring fecal consistency as follows: $0=-$ solid; $1=$ pasty; $2=$ semi-liquid; $3=$ liquid, with scores of 2 or more considered diarrheic. Calves \#1 (PDCoV) and \#5 (PEDV) were monitored for long-term clinical signs and virus shedding until post-inoculation day (PID) 16-17. The other inoculated or mock-infected calves were kept for short-term studies and were euthanized for histopathological examination at acute to mild stages (PIDs 3, 8 or 9) of viral infection (Table 1).

Rectal and nasal swabs or serum samples were collected and prepared as described previously [4, 6]. Rectal and nasal swabs were diluted 1:10 and 1:50, respectively, in MEM. Virus RNA was extracted using the Mag-MAX Viral RNA Isolation Kit (Applied Biosystems, Foster City, CA, USA) according to the manufacturer's instructions. Titers of virus shed in feces were determined by qRT-PCR using the OneStep RT-PCR Kit (QIAGEN, Valencia, CA, USA) $[6,8]$. The detection limit of qRT-PCR for PDCoV was $10 \mathrm{GE}$ per reaction, corresponding to 5.3, 4.6, and 3.6 $\log _{10} \mathrm{GE} / \mathrm{ml}$ of PDCoV in nasal, rectal swab, and serum samples, respectively [8]. The detection limit of qRT-PCR for PEDV was $10 \mathrm{GE}$ per reaction, corresponding to 4.8 $\log _{10} \mathrm{GE} / \mathrm{ml}$ of PEDV in rectal swab samples [6].

PDCoV OH-FD22-P5 (passage five) and PEDV PC22P40 (passage 40) viruses, grown on LLC-PK or Vero cells, respectively, were used to infect LLC-PK or Vero cells, respectively, in 96-well plates. The viral antigens expressed were detected by indirect immunofluorescence assay (IFA) [2, 3, 12]. A multiplicity of infection of 0.1 was used for each viral inoculation in LLC-PK and Vero cells treated with 5 and $10 \mu \mathrm{g} / \mathrm{ml}$ of trypsin, respectively $[2,3,12]$. The cell culture conditions used to infect LLC-PK or Vero cells with each virus were described previously [2, 3, 12]. Characteristic cytopathic effects (CPE) [2, 3, 12] were monitored regularly in inoculated LLC-PK or Vero cells. When CPE was pronounced, PDCoV-inoculated LLC-PK (PID 1) and PEDV-inoculated Vero cells (PID 2) were fixed with $100 \%$ ethanol at $4^{\circ} \mathrm{C}$ overnight for IFA. The fixed cells were washed with $0.01 \mathrm{M}$ phosphate buffered saline (PBS) ( $\mathrm{pH}$ 7.4). Blocking was performed with $1 \times$ buffered solution of casein $\left(10 \times\right.$ Power Block ${ }^{\mathrm{TM}}$ Universal Blocking Reagent, Biogenx, CA, USA) in distilled water for $1 \mathrm{hr}$ at room temperature. Four-fold serial dilutions, starting at 1:4, of the paired serum samples of calves \#1 and \#5, obtained at PID 0 and PID 16-17 were added to the wells and the plates were incubated overnight. Swine hyperimmune antiserum against PDCoV (1:100) or monoclonal antibody against the spike (S) protein of PEDV (6C8-1) (1:200), with the appropriate detection antibodies, were added to the wells as IFA positive controls [2, 3, 12]. Goat polyclonal antibodies specific for bovine, swine or mouse IgG (whole IgG) conjugated to fluorescein (KPL, Gaithersburg, MD, USA) were diluted $1: 100$ in $0.01 \mathrm{M}$ PBS. The plates were incubated for $1 \mathrm{hr}$ at $37^{\circ} \mathrm{C}$. The stained plates were evaluated using a fluorescence microscope. The antibody titers were expressed as the reciprocal of the highest serum dilution in the wells that were positive for PDCoV or PEDV antigen detection.

Formalin-fixed or frozen small (duodenum, jejunum and ileum) and large (cecum/colon) intestinal tissues and other major organs were examined macroscopically and histologically and tested by IF for PDCoV or PEDV antigens $[5,6,8]$. Tissues from the negative control calf (\#8) were tested for histological comparisons and as an IF negative control. Tissues from PDCoV- or PEDV-infected Gn pigs at PID 3 were also tested as positive controls $[6,8]$.

Clinical observations revealed that none of the $\mathrm{Gn}$ calves inoculated with PDCoV (calves \#1-4), PEDV (calves \#5-7), or mock (calf \#8) exhibited diarrhea or other clinical signs throughout the course of the experiment (Fig. 1). PDCoV RNA was first detected in fecal samples at PID 2 (calves \#1 and 2) or PID 3 (calves \#3 and 4) by qRTPCR (Table 1). Fecal PDCoV RNA titers, ranging from 7.2 to $8.4 \log _{10} \mathrm{GE} / \mathrm{ml}$, peaked at PID 2 (calf \#2), PID 3 (calf \#1), or PID 4-6 (calf \#3) (Table 1). Calf \#1, was followedup long-term and had prolonged viral RNA shedding until PID 16 (Fig. 1). The highest fecal PDCoV RNA titer of calf \#1 was at PID $3\left(8.4 \log _{10} \mathrm{GE} / \mathrm{ml}\right)$, and the titers decreased progressively thereafter (Fig. 1). On the other hand, none of the Gn calves inoculated with PEDV shed detectable PEDV RNA in the feces at PIDs 1-9 (calves \#6 and \#7) and PIDs 1-17 (calf \#5). The negative control (calf \#8) did not shed detectable PDCoV or PEDV RNA in the 


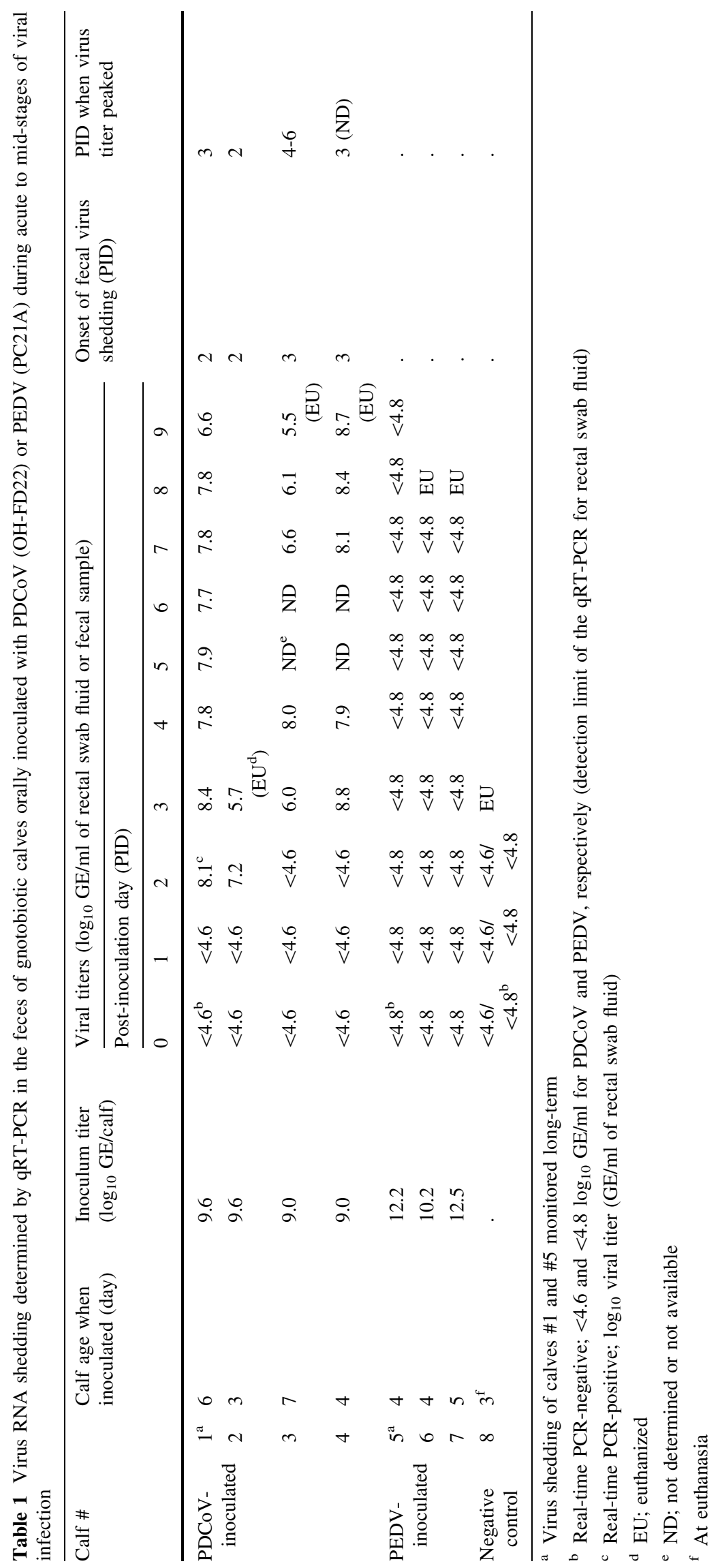


Fig. 1 Persisting fecal viral RNA shedding of calf \#1 inoculated with $\mathrm{PDCoV}$ strain OH-FD22-P5, but without diarrhea. Calf \#1 was inoculated orally with $9.6 \log _{10} \mathrm{GE}$ of the gnotobiotic pig-passaged $\mathrm{OH}-$ FD22-P5. It was monitored for long-term clinical signs and virus shedding at PIDs 1 to 16 . Rectal swabs were collected daily throughout the experiment. The PDCoV fecal shedding titers were determined by qRT-PCR. The dotted line indicates the detection limit (4.6 $\log _{10} \mathrm{GE} / \mathrm{ml}$ ) of the qRT-PCR

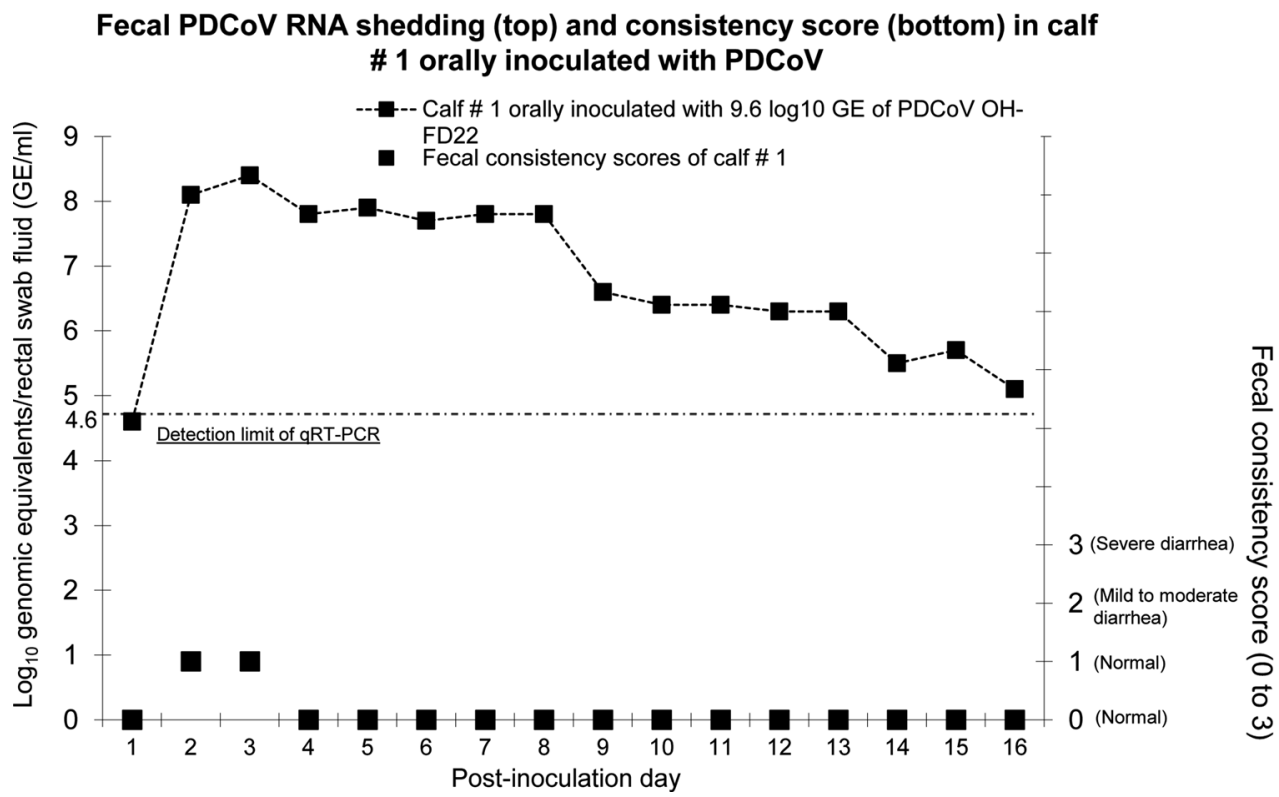

feces during the experiment. Nasal swab or serum samples collected from PDCoV-inoculated calf \#1 were also tested by qRT-PCR. Serum samples were collected at PIDs 3 and 15, and nasal swab samples were collected at PIDs 2, 6, 9, 13, and 16. No PDCoV RNA was detected in the serum $\left(<3.6 \log _{10} \mathrm{GE} / \mathrm{ml}\right)$ and nasal swab $\left(<5.3 \log _{10} \mathrm{GE} / \mathrm{ml}\right)$ samples tested at the time-points indicated.

IFA showed that calf \#1 had serum IgG antibodies (titer of 1,024) against PDCoV OH-FD22 at PID 16, indicating seroconversion. Large numbers of IF-stained cells were consistently observed when PDCoV-infected LLC-PK cells were incubated with the serum diluted $1: 4$ to $1: 1,024$ (Fig. 2). No PDCoV-specific IgG antibodies were detected in the prebled serum samples of calf \#1 and negative control calf \#8 (Fig. 2). In PEDV-inoculated calf \#5, there were no detectable PEDV-specific IgG antibodies in the serum samples at PID 0 and PID 17 (Fig. 2). Light microscopy analysis revealed that none of the PDCoV- or PEDV-inoculated calves had major histological changes in the intestine. Additionally, by IF, no PDCoV or PEDV antigen-positive cells were observed in the small and large intestinal tissues of PDCoV or PEDV-inoculated Gn calves, while the intestinal tissues (mainly villous enterocytes) of PDCoV or PEDVinfected Gn pigs were found to be positive for PDCoV or PEDV antigen in earlier studies [6, 8].

Our study demonstrated that Gn calves orally inoculated with the PDCoV strain OH-FD22 (ICs of cell-culture grown PDCoV from infected $\mathrm{Gn}$ pigs) develop an acute infection with persistent fecal PDCoV RNA shedding and PDCoVspecific serum IgG antibody responses, but show no signs of significant intestinal lesions or clinical disease [8]. These observations are similar to a previous report showing that, human SARS-CoV strain Urbani, prior to its adaptation to mice (MA105 strain), replicated in the lungs of young mice, as indicated by their lung homogenates testing positive by qRTPCR. However, the Urbani strain-inoculated mice did not show any clinical signs, but only mild histological lesions with low expression of viral antigens [13]. In our study, there were no detectable fecal viral RNA shedding, virus-specific serum IgG antibody responses, histological lesions, and clinical disease in Gn calves orally inoculated with the PEDV strain PC21A (ICs of wild-type PEDV-infected Gn pigs) [6]. For the past 40 years, PEDV has been found only within pigs, indicating that PEDV may have only adapted to pigs [9]. In contrast, PDCoV may not yet be completely adapted to pigs, and the disease caused by PDCoV has emerged only 2-3 years ago. PDCoV-infected pigs appeared to shed less PDCoV RNA and had a lower peak of viral titer in the feces, compared with experimental PEDV infections [7, 11], suggesting that there is a lower replication of $\mathrm{PDCoV}$ in the intestine of pigs, possibly due to its incomplete adaptation to pigs.

In a molecular surveillance study in China and Hong Kong conducted in 2007-2011, deltacoronaviruses (DCoVs) were detected only in pigs and wild birds [18]. However, DCoVs were detected previously in rectal swabs of small mammals, such as Asian leopard cats and Chinese ferret badgers, in live-animal markets in China, in 2005-2006 [1]. The helicase and $\mathrm{S}$ genes of the CoVs isolated from the wild small mammals were closely related to those of PDCoV [18], suggesting that a potential interspecies transmission event may have occurred leading to DCoV transmission between wild small mammals and pigs. However, further studies are needed to define the potential role of small mammals as an intermediate host of PDCoV and the mechanisms of interspecies transmission of DCoVs between small mammals and domestic pigs or wild birds. Our data also support the 


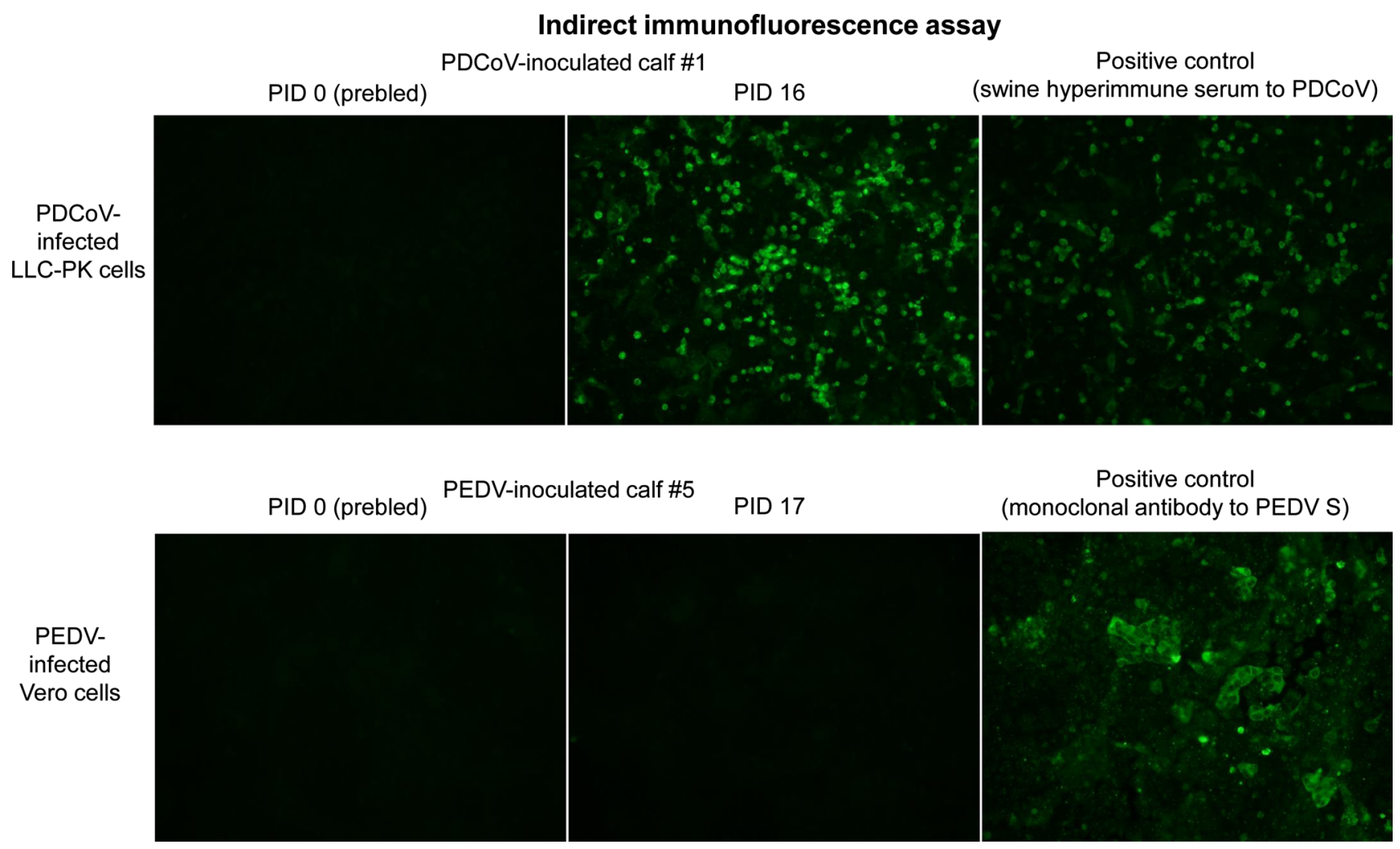

Fig. 2 Detection of serum IgG antibodies against PDCoV or PEDV by indirect immunofluorescence assay (IFA). PDCoV-inoculated calf $\# 1$ and PEDV-inoculated calf \#5 were monitored for long-term clinical signs and virus shedding until PID 16-17. The serum samples were collected at PID 0 and PID 16-17. Four-fold serial dilutions, beginning at 1:4, of the paired serum samples of calves \#1 and \#5 were added and incubated onto PDCoV-infected LLC-PK cells or PEDV-infected Vero cells. Swine hyperimmune antiserum against

potential ability of the newly emerged PDCoV to infect another animal species, such as cattle [16]. However, our study did not demonstrate PDCoV's ability of direct interspecies transmission between pigs and cattle.

The $\mathrm{S}$ protein of $\mathrm{CoVs}$ is critical for regulating interactions with specific host cell receptor glycoproteins to mediate viral entry [15]. Based on our data, it is possible that interactions between PDCoV S protein and the host cell receptor or co-receptor binding may occur in the bovine intestine, whereas this may not be the case for PEDV S protein. The major cellular receptors of PEDV and $\mathrm{PDCoV}$ in pigs are unknown $[10,14]$. The CoV S proteins can be divided into two functional subunits, $\mathrm{S} 1$ and $\mathrm{S} 2$. The $\mathrm{S} 1$ is involved in the receptor recognition and binding via the N-terminal or C-terminal virus receptor binding domains. Therefore, proteolysis between $\mathrm{S} 1$ and $\mathrm{S} 2$ is a critical determinant for $\mathrm{CoV}$ tropism and pathogenesis. The $\mathrm{CoV} \mathrm{S}$ proteins can be cleaved by different animal proteases [15]. The extent of cleavage of PEDV and PDCoV S proteins might differ in the bovine intestine, possibly depending on their adaptation to the bovine intestinal
PDCoV or monoclonal antibody against the spike protein of PEDV was added as positive controls. Note that large numbers of IF-stained cells were evident when PDCoV-infected LLC-PK cells were incubated with the serum of calf \#1 diluted 1:4. In PEDV-inoculated calf \#5, however, there were no detectable PEDV-specific IgG antibodies in the serum samples (diluted 1:4) at PID 0 and PID 16. Original magnification, all $\times 200$

environment; for example, exogenous proteolytic enzymes of bovine origin may influence PEDV or PDCoV S proteinhost cell receptor binding. However, these assumptions should be verified by further studies.

In our study, PDCoV-inoculated calves showed acute fecal viral RNA shedding, followed by progressively decreased titers thereafter. There were also no PDCoV RNApositive nasal or serum samples from PDCoV-inoculated calf \#1, indicating that PDCoV infection may be limited to the intestine, similar to PDCoV infections in pigs [10], although PDCoV-infected pigs also showed viremia (viral RNA in serum) [10]. Our study did not identify which type of cells in the intestines of inoculated calves could be the target for PDCoV replication, resulting in fecal shedding. Our IF staining experiments identified PDCoV antigen-positive cells in intestinal tissues from PDCoV-infected Gn pigs used as positive controls. Similar to the GIII.2 bovine norovirus (CV186-OH strain) that was shown to cause fecal virus RNA shedding in infected Gn calves, but in the absence of histological lesions or viral antigen/RNA in the intestine [5], $\mathrm{PDCoV}$ did not induce major histological changes in the 
intestine of Gn calves, such as necrosis of intestinal epithelium or villous atrophy. This result is also in line with data showing no PDCoV-positive cells in the intestinal epithelium. Based on these observations, PDCoV may have infected the intestine of calves as an attenuated virus that does not cause clinical disease and histological lesions, with low levels of viral antigen detected in enterocytes in inoculated animals. Nevertheless, our study did not clearly rule out an abortive infection with limited PDCoV replication in calves.

In addition, interestingly, no PEDV-specific IgG antibodies were detected by IFA in the serum of PEDV-inoculated calf \#5 at PID 16. However, a virus neutralization (VN) assay using a PEDV strain Iowa106 [3, 12], showed low PEDV neutralizing activity in the sera of calf \#5 at PID 16 and prebled prior to PEDV inoculation, with VN titers of $4^{2.8}$ (PID 16) and $4^{2.5}$ (prebled). Additional studies using larger numbers of serum samples are needed to confirm whether bovine sera have PEDV antibodies or neutralizing substances.

Collectively, our data indicate that calves are susceptible to infection with the newly emerged PDCoV, but not with the swine enteric CoV, PEDV. However, the infectivity of PDCoV in calves was limited, as manifested by no clinical disease and histological lesions, possibly due to its incomplete adaptation to the bovine intestine. Other animal species with frequent contacts with pigs, such as poultry, also need to be monitored for potential infectivity by PDCoV.

\section{Compliance with ethical standards}

Funding We thank Dr. Juliette Hanson, Ronna Wood, and Jeffery Ogg for assistance with animal care; and Xiaohong Wang and Marcia Lee for technical assistance. Salaries and research support were provided by state and federal funds appropriated to the Ohio Agricultural Research and Development Center, The Ohio State University.

Ethical approval All applicable international, national, and/or institutional guidelines for the care and use of animals were followed.

Conflict of interest Neither of the authors of this paper has a financial or personal relationship with other people or organizations that could inappropriately influence or bias the content of the paper. All authors have seen and approved the manuscript.

\section{References}

1. Dong BQ, Liu W, Fan XH, Vijaykrishna D, Tang XC, Gao F, Li LF, Li GJ, Zhang JX, Yang LQ, Poon LL, Zhang SY, Peiris JS, Smith GJ, Chen H, Guan Y (2007) Detection of a novel and highly divergent coronavirus from asian leopard cats and Chinese ferret badgers in Southern China. J Virol 81:6920-6926

2. Hu H, Jung K, Vlasova AN, Chepngeno J, Lu Z, Wang Q, Saif LJ (2015) Isolation and characterization of porcine deltacoronavirus from pigs with diarrhea in the United States. J Clin Microbiol 53:1537-1548

3. Hu H, Jung K, Vlasova AN, Saif LJ (2016) Experimental infection of gnotobiotic pigs with the cell-culture-adapted porcine deltacoronavirus strain OH-FD22. Arch Virol 161:3421-3434

4. Jung K, Alekseev KP, Zhang X, Cheon DS, Vlasova AN, Saif LJ (2007) Altered pathogenesis of porcine respiratory coronavirus in pigs due to immunosuppressive effects of dexamethasone: implications for corticosteroid use in treatment of severe acute respiratory syndrome coronavirus. J Virol 81:13681-13693

5. Jung K, Scheuer KA, Zhang Z, Wang Q, Saif LJ (2014) Pathogenesis of GIII.2 bovine norovirus, CV186-OH/00/US strain in gnotobiotic calves. Vet Microbiol 168:202-207

6. Jung K, Wang Q, Scheuer KA, Lu Z, Zhang Y, Saif LJ (2014) Pathology of US porcine epidemic diarrhea virus strain PC21A in gnotobiotic pigs. Emerg Infect Dis 20:662-665

7. Jung K, Annamalai T, Lu Z, Saif LJ (2015) Comparative pathogenesis of US porcine epidemic diarrhea virus (PEDV) strain PC21A in conventional 9-day-old nursing piglets vs. 26-day-old weaned pigs. Vet Microbiol 178:31-40

8. Jung K, Hu H, Eyerly B, Lu Z, Chepngeno J, Saif LJ (2015) Pathogenicity of 2 porcine deltacoronavirus strains in gnotobiotic pigs. Emerg Infect Dis 21:650-654

9. Jung K, Saif LJ (2015) Porcine epidemic diarrhea virus infection: Etiology, epidemiology, pathogenesis and immunoprophylaxis. Vet J 204:134-143

10. Jung K, Hu H, Saif LJ (2016) Porcine deltacoronavirus infection: Etiology, cell culture for virus isolation and propagation, molecular epidemiology and pathogenesis. Virus Res 226:50-59

11. Madson DM, Arruda PH, Magstadt DR, Burrough ER, Hoang H, Sun D, Bower LP, Bhandari M, Gauger PC, Stevenson GW, Wilberts BL, Wang C, Zhang J, Yoon KJ (2015) Characterization of Porcine Epidemic Diarrhea Virus Isolate US/Iowa/18984/2013 Infection in 1-Day-Old Cesarean-Derived Colostrum-Deprived Piglets. Vet Pathol 53:44-52

12. Oka T, Saif LJ, Marthaler D, Esseili MA, Meulia T, Lin CM, Vlasova AN, Jung K, Zhang Y, Wang Q (2014) Cell culture isolation and sequence analysis of genetically diverse US porcine epidemic diarrhea virus strains including a novel strain with a large deletion in the spike gene. Vet Microbiol 173:258-269

13. Roberts A, Deming D, Paddock CD, Cheng A, Yount B, Vogel L, Herman BD, Sheahan T, Heise M, Genrich GL, Zaki SR, Baric R, Subbarao K (2007) A mouse-adapted SARS-coronavirus causes disease and mortality in BALB/c mice. PLoS Pathog 3:e5

14. Shirato K, Maejima M, Islam MT, Miyazaki A, Kawase M, Matsuyama S, Taguchi F (2016) Porcine aminopeptidase $\mathrm{N}$ is not a cellular receptor of porcine epidemic diarrhoea virus, but promotes its infectivity via aminopeptidase activity. J Gen Virol 97:2528-2539

15. Simmons G, Zmora P, Gierer S, Heurich A, Pohlmann S (2013) Proteolytic activation of the SARS-coronavirus spike protein: cutting enzymes at the cutting edge of antiviral research. Antiviral Res 100:605-614

16. Su S, Wong G, Shi W, Liu J, Lai AC, Zhou J, Liu W, Bi Y, Gao GF (2016) Epidemiology, Genetic Recombination, and Pathogenesis of Coronaviruses. Trends Microbiol 24:490-502

17. Wang L, Byrum B, Zhang Y (2014) Detection and genetic characterization of deltacoronavirus in pigs, Ohio, USA, 2014. Emerg Infect Dis 20:1227-1230

18. Woo PC, Lau SK, Lam CS, Lau CC, Tsang AK, Lau JH, Bai R, Teng JL, Tsang CC, Wang M, Zheng BJ, Chan KH, Yuen KY (2012) Discovery of seven novel Mammalian and avian coronaviruses in the genus deltacoronavirus supports bat coronaviruses as the gene source of alphacoronavirus and betacoronavirus and avian coronaviruses as the gene source of gammacoronavirus and deltacoronavirus. J Virol 86:3995-4008 